\title{
Impact de la micro pesanteur sur le flux de chaleur et la température de remouillage lors de la mise en froid d'un tube : application au rallumage de moteurs-fusée
}

\author{
Brian Verthier ${ }^{1,2,4, a}$, Gian Piero Celata ${ }^{3}$, Giuseppe Zummo ${ }^{3}$, Catherine Colin ${ }^{1,2}$ \\ ET JÉRÉMY FOLLET ${ }^{4}$ \\ 1 Université de Toulouse, INPT, UPS, IMFT (Institut de Mécanique des Fluides de Toulouse), Allée Camille Soula, \\ 31400 Toulouse, France \\ 2 CNRS, IMFT, 31400 Toulouse, France \\ 3 ENEA, Institute for Thermal Fluid Dynamics, Via Anguillarese 301, 00060 S.M. Galeria, Rome, Italy \\ 4 SNECMA, Forêt de Vernon, BP802, 27208 Vernon Cedex, France
}

Reçu le 20 avril 2009

\begin{abstract}
Résumé - Cet article décrit la stratégie expérimentale développée afin d'améliorer la modélisation des écoulements diphasiques liquide-vapeur présents lors de la mise en froid de moteurs-fusée par des fluides cryogéniques en micro pesanteur. Une analyse de similitude est faite afin de déterminer les nombres adimensionnels pertinents pour le dimensionnement d'une expérience similaire à ces écoulements. Une analyse bibliographique sur les expériences menées en micro pesanteur, la température de remouillage et le flux lors de l'ébullition en film montre le manque de modèles adaptés à la micro gravité. Des résultats de « quenching » d'un tube de pyrex par du FC72 lors de vols paraboliques sont présentés, en particulier l'impact du niveau de gravité et du sous refroidissement sur la température de remouillage et le flux lors de l'ébullition en film. Les résultats montrent une baisse du transfert thermique en micro pesanteur et une augmentation de la température de remouillage.
\end{abstract}

Mots clés : Trempe / micro pesanteur / ébullition en film / remouillage / transfert thermique

Abstract - Effect of reduced gravity on heat transfer and rewetting temperature during quenching: application to rocket engines reignition. This paper describes the experimental strategy developed to improve the modeling of liquid-vapor flows during the chill down of rocket engines by cryogenic fluid in microgravity. A similarity analysis is performed to determine the relevant dimensionless numbers for the design of an experiment similar to engine flows. A literature review on reduced gravity quenching experiments, and on rewetting temperature and film boiling heat transfer shows the lack of validated models for microgravity. Experimental results obtained with the quenching of a glass tube by FC72 during parabolic flight are presented. Especially the impact of gravity and subcooling on rewetting temperature and film boiling heat transfer is investigated. Results show an increase in rewetting temperature, and a decrease in film boiling heat transfer under reduced gravity in agreement with the literature. The comparison of $0 g$ flow pattern with corresponding tests on ground point out a behavior at $0 g$ closer to $1 g$ upflow than $1 g$ downflow.

Key words: Quenching / microgravity / film boiling / rewetting / chilldown / heat transfer

\section{Introduction}

En micro pesanteur, de nombreux processus impliquent des écoulements diphasiques et des transferts thermiques par ébullition. Dans les moteurs-fusée cryogéniques, les lignes d'alimentation et les canaux des turbopompes doivent être refroidis avant l'allumage. Dans

\footnotetext{
${ }^{a}$ Auteur pour correspondance : verthier@imft.fr
}

le cas d'allumages multiples, cela peut être fait en micro pesanteur. Une stratégie a été établie afin de traiter ce sujet et d'obtenir une modélisation fiable de la mise en froid de moteurs-fusée cryogéniques en gravité variable. Les activités menées sont supportées et dirigées techniquement par SNECMA moteur et le Centre National d'Etudes Spatiales (CNES). Celles-ci incluent des analyses théoriques, des expériences de validation et le 


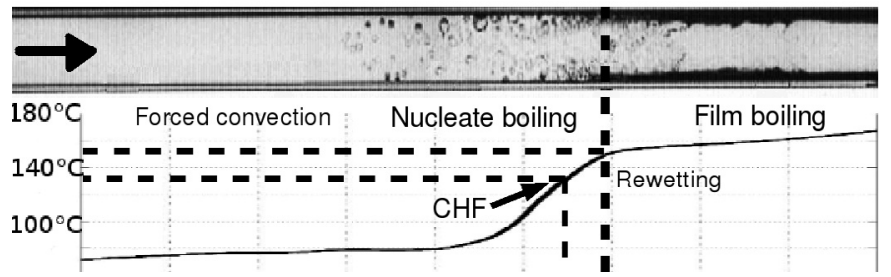

Fig. 1. Évolution du régime d'écoulement (en haut) et de la température paroi (en bas) en micro pesanteur lors de la trempe d'un tube.

développement de codes. Au cours de ce refroidissement, les tuyauteries sont ramenées de $300 \mathrm{~K}$ à des températures cryogéniques par des écoulements d'ergols. Au commencement, la température de la paroi est suffisamment élevée pour maintenir de l'ébullition en film, jusqu'à ce que celleci soit assez refroidie pour être remouillée, et un régime d'ébullition nucléée est alors observé (Fig. 1).

Ce processus, appelé «quenching », mise en froid, ou trempe, est caractérisé par le parcours de toute la courbe d'ébullition en chaque point de la paroi. La modélisation précise de la trempe permet de prédire la durée de la mise en froid et donc la consommation d'ergols nécessaire. Les codes de calculs existants ne sont pas validés en micro pesanteur et les lois de fermetures pour l'ébullition de fluides cryogéniques en gravité variable n'ont pas été déterminées. En raison du manque de résultats précis dans la littérature (Sect. 2), des expériences doivent être menées afin de construire les modèles adéquats. Pour des raisons pratiques, celles-ci seront menées avec un fluide de similitude (Sect. 3).

\section{Contexte expérimental et état de l'art}

Le phénomène de mise en froid, ou « quenching », peut être défini par le refroidissement d'une surface surchauffée par un écoulement liquide, bien que ce terme soit utilisé dans de nombreuses configurations. En effet, la surface peut être une sphère, un cylindre, une plaque, et le liquide peut se présenter sous forme d'une goutte, d'un jet impactant, ou d'un écoulement.

Ainsi, de nombreuses études ont été menées sur la trempe pour des applications en sidérurgie, concernant le refroidissement des plaques d'acier sortant des laminoirs. La trempe d'objets surchauffés a également été l'objet d'investigations, en raison des applications pour les traitements thermiques de métaux [1]. Le remouillage de conduites surchauffées a été étudié notamment pour modéliser le comportement de réacteurs à eau pressurisée en cas de brèche du circuit hydraulique primaire [2].

Ce n'est que récemment, avec l'essor des technologies spatiales, que la trempe en gravité réduite est devenue un sujet d'étude majeur [3]. Dans ce cas précis, la complexité de l'usage de fluide cryogénique s'ajoute à celle de la trempe et de la gravité réduite.

Dans la littérature, ces configurations sont souvent confondues. En général, le terme Leidenfrost décrit l'impact d'une goutte sur une surface isotherme. La température minimum de film stable s'applique à la transition vers l'ébullition en film lors de l'ébullition en vase, et la trempe s'utilise souvent pour la trempe d'objets dans un liquide au repos. Ces configurations sont toutes différentes, mais ont comme points communs de présenter de l'ébullition en film et nucléée.

Ainsi, les corrélations et modèles existants étant basés sur une expérience donnée, pour une application précise, leur usage doit être fait avec prudence, bien que le phénomène local reste le même, tout comme les paramètres influents. Comme nous nous concentrons sur le refroidissement de moteurs-fusée, le premier régime observé est l'ébullition en film. Celui-ci est caractérisé par un faible échange thermique et va donc grandement influencer la durée de la mise en froid. Ainsi, nous nous limiterons ici à l'étude de ce régime et à la transition vers l'ébullition nucléée, la température de remouillage. Le flux critique et l'ébullition nucléée feront l'objet d'une étude postérieure.

\subsection{Flux lors de l'ébullition en film}

Bromley [4], en effectuant un bilan entre forces de pesanteur et de frottement, établit une des premières corrélations sur le coefficient d'échange lors de l'ébullition en film en vase, pour des cylindres et des plaques, en gravité terrestre. Cette corrélation dépend majoritairement de la gravité $g$, de la surchauffe paroi $T_{w}-T_{s}$, et des propriétés du fluide.

$$
\begin{aligned}
& h_{\text {con }}=0,62 \\
& \quad \times\left[\frac{\lambda_{2}^{3} g_{0} \rho_{2}\left(\rho_{1}-\rho_{2}\right)\left(\Delta h_{1,2}+0.4 C p_{2}\left(T_{w}-T_{s}\right)\right.}{\mu_{2}\left(T_{w}-T_{s}\right) D}\right]^{1 / 4}
\end{aligned}
$$

Breen et al. [5] proposèrent une corrélation du même type, mais pour des fluides cryogéniques. En 1953, Bromley [6] suggéra pour des cas de convection forcée une modification de l'équation (1). De nombreux auteurs ont également proposé certains ajustements, par la prise en compte au lieu d'une longueur caractéristique $D$ d'une longueur capillaire [7] ou d'une longueur d'onde de l'instabilité de Kevin-Helmholtz de l'interface liquide-vapeur [8]. Frederking et al. [9] proposèrent une corrélation basée sur un niveau d'accélération local au lieu de la gravité terrestre pour la trempe de sphère, et plus récemment, certains modèles d'ébullition en film convective ont été développés [10], avec une approche locale, en prenant en compte le taux de vide $\alpha_{2}$, ou le titre massique $x_{2}$. Ainsi, Hammouda et al. [11] établirent un modèle local offrant une meilleure prédiction des expériences menées que les corrélations présentées plus tôt. Ces modèles sont plus difficilement applicables en raison du besoin de connaître des paramètres locaux.

Si les modèles présentés ci-dessus offrent une bonne prédiction des données obtenues au sol, l'extrapolation directe à la micro pesanteur est impossible. Peu d'études ont été menées sur l'impact de la gravité réduite. 
Westbye et al. [12] observèrent une réduction des transferts thermiques lors de la trempe d'un tube d'acier en micro pesanteur, en comparaison avec la configuration horizontale. Au contraire, Kawanami et al. [13] pour la trempe de tubes de pyrex verticaux par du LN2 conclurent sur une amélioration des transferts thermiques en $0 \mathrm{~g}$. Cette contradiction pourrait venir de l'orientation différente en $1 g$, ou du fait que Kawanami initialise la trempe avant la période de micro pesanteur. L'étude du remouillage d'une plaque en $0 g$ menée par Xu [14] montre une baisse du transfert thermique lors de l'ébullition en film. Il compara ses résultats avec les corrélations présentées plus haut et observa que seule la corrélation basée sur la dominance des effets inertiels de Bromley [6] pouvait donner un ordre de grandeur du résultat, pour une vitesse du liquide supérieure à 0,36 m.s ${ }^{-1}$. En 1996, Kawaji [15] publia une revue des expériences de «quenching » menées en micro pesanteur, et établit que la plupart d'entre elles conclut sur une diminution du transfert thermique.

\subsection{Température de remouillage}

De nombreuses études ont été menées sur la transition entre l'ébullition en film et l'ébullition nucléée. Spiegler et al. [16] ont proposé, en se basant sur les équations de Van der Walls, que la température de remouillage lors de l'effet Leidenfrost est égale à $\frac{27}{32} T_{c}$, où $T_{c}$ est la température critique. Semeria et al. [17] ajoutèrent à ce terme l'impact des propriétés de la paroi pour un cas non isotherme, et Baumsteiner et al. [18] ajoutèrent l'impact de la température de la goutte. Ce premier type de corrélation considère que le remouillage est donc majoritairement dépendant des propriétés de la paroi et du fluide.

Un second type de corrélation considère que celleci est majoritairement fonction de l'écoulement. Ainsi, Berenson et al. [7] ont établi une corrélation valable en ébullition en vase basée sur instabilité de Taylor de l'interface. Henry [17] proposa une modification en prenant en compte le sous refroidissement du liquide, et Iloeje [2] quantifia l'impact du débit liquide.

Plus récemment, De Salve et al. [19] combina l'impact prédit par les deux types de corrélations présentées ci-dessus. Ils prirent en comptel'impact de la paroi et des propriétés physiques des premières corrélations, et de l'écoulement des deuxièmes (Éq. (2)). Ils observèrent un bon accord entre la corrélation établie et les résultats expérimentaux pour des faibles débits et Carbajo [20] proposa une modification du terme de débit pour les fortes valeurs. Récemment, de nombreux modèles locaux ont été développés [21,22], basés sur des titres massiques et taux de vide.

$$
\begin{aligned}
T_{\text {rew }}=T_{1}+0,29 & \left(\frac{27}{32} T_{c}-T_{1}\right)\left(1+0,279 G^{0,49}\right) \\
& \times\left[\mathrm{e}^{\frac{3,06 * 10^{6}}{\lambda C_{p} \mid w}} \operatorname{erfc}\left(\frac{1751,5}{\sqrt{\left.\lambda \rho C_{p}\right|_{w}}}\right)\right]^{-1}
\end{aligned}
$$

L'ensemble des corrélations présentées ici a été basé sur des expériences menées en gravité terrestre, et est donc inapplicable dans notre cas. A notre connaissance, aucune corrélation n'existe pour la trempe d'un tube en gravité réduite. Quantitativement, Xu [14] et AdhamKhodaparast et al. [23] ont observé une diminution de la température de remouillage en $0 g$ lors de la trempe d'une plaque horizontale. Westbye et al. [12] ont obtenu la même conclusion pour un tube d'acier horizontal, et Celata et al. [24] pour un tube en pyrex vertical.

\section{Critères de similitude entre deux écoulements lors du phénomène de trempe}

Deux expériences sont similaires quand leurs nombres adimensionnels caractéristiques sont égaux. Pour obtenir ces nombres, une méthode consiste en l'adimensionnalisation des équations décrivant le phénomène. Pour la trempe, celles-ci sont données par des lois classiques de conservation mono dimensionnelle de la masse, de la quantité de mouvement et de l'enthalpie (Éqs. (3, 4, 5) respectivement). Le couplage avec la paroi peut être pris en compte par un bilan d'énergie (Éq. (6)). Équation (3) est la conservation de la masse intégrée dans une section de tube et $\alpha_{k}, \rho_{k}$, et $x_{k}$ sont la fraction volumique, la densité et le titre massique de la phase $k$, avec $k=1$ pour le liquide, et $k=2$ pour sa vapeur. $G$ est le débit massique surfacique en $\mathrm{kg} \cdot \mathrm{m}^{2} . \mathrm{s}^{-1}$, et $z$ la direction de l'écoulement, soit l'axe du tube. Le troisième terme de l'équation (3) représente le transfert de masse à l'interface.

$$
\frac{\partial \alpha_{k} \rho_{k}}{\partial t}+G \frac{\partial \alpha_{k} x_{k}}{\partial z}-G \frac{\partial x_{k}}{\partial z}=0
$$

L'équation (4) est la conservation de la quantité de mouvement, projetée sur l'axe $z$, où $V_{i}$ est la vitesse de l'interface, $p_{k}$ la pression moyenne, $g$ l'accélération locale égale à $9,81 \mathrm{~m} . \mathrm{s}^{2}$ sur terre, et $\tau_{w, k}$ le taux de frottement pariétal sur le périmètre $P_{w, k} \cdot \tau_{i, k}$ et $P_{i, k}$ représentent le frottement interfacial et le périmètre interfacial, $A$ la section de passage et $D$ le diamètre du tube. En négligeant les effets capillaires, le bilan interfacial nous fournit juste le raccord des frottements à l'interface $\tau_{i, 1}+\tau_{i, 2}=0$.

$$
\begin{array}{r}
G \frac{\partial x_{k}}{\partial t}+\frac{G^{2}}{\rho_{k}} \frac{\partial x_{k}^{2} / \alpha_{k}}{\partial z}-G V_{i} \frac{\partial x_{k}}{\partial z}+\alpha_{k} \frac{\partial p_{k}}{\partial z}-\alpha_{k} \rho_{k} g \\
-\tau_{w, k} \frac{P_{w, k}}{A}-\tau_{i, k} \frac{P_{i, k}}{A}=0
\end{array}
$$

L'équation de conservation de l'enthalpie totale $h_{k}$ est donnée par (5), où $q_{w, k}$ et $q_{i, k}$ représentent les transferts de chaleur à la paroi et à l'interface respectivement. Le bilan interfacial nous conduit à $q_{i, 1}+q_{i, 2}=G \Delta h_{1,2} \frac{A}{P_{i}} \frac{\partial x}{\partial z}$.

$$
\begin{array}{r}
\rho_{k} \frac{\partial h_{k} \alpha_{k}}{\partial t}+G \frac{\partial h_{k} x_{k}}{\partial z}-G h_{k, s a t} \frac{\partial x_{k}}{\partial z}+\alpha_{k} \frac{\partial p_{k}}{\partial t}-G x_{k} g \\
-q_{w, k} \frac{P_{w, k}}{A}-q_{i, k} \frac{P_{i, k}}{A}=0
\end{array}
$$


En négligeant l'énergie apportée nécessaire à la surchauffe du gaz par rapport à l'énergie utilisée pour la vaporisation, le bilan d'énergie dans une section peut être donné par équation (6), où l'indice $w$ indique une propriété de la paroi, $\overline{T_{w}}$ sa température moyenne et $C p_{w}$ sa chaleur spécifique.

$$
\begin{aligned}
& \sum_{k=1,2} \frac{q_{w, k} P_{w, k}}{A}=\frac{\rho_{w} A_{w} C p_{w}}{A} \frac{\partial \overline{T_{w}}}{\partial t}= \\
& G\left(\Delta h_{1,2}+C p_{1}\left(T_{s}-T_{1}\right)\right) \frac{\partial x_{2}}{\partial z}+G x_{1} \frac{\partial T_{1}}{\partial z}
\end{aligned}
$$

Les nombres adimensionnels caractéristiques sont obtenus par les formes adimensionnelles des équations (3), (4) et (5), en normant chaque variable par une grandeur caractéristique, la difficulté réside donc dans le choix de cette normalisation. Par exemple, la vitesse caractéristique de chaque phase dépend fortement du régime d'écoulement. Ainsi, arbitrairement, et comme nous ne pouvons imposer que des paramètres d'entrée, le diamètre $D$ est choisi comme échelle de longueur, $V_{k}=G / \rho_{k}$ comme échelle de vitesse de la phase $k$ et $\Theta$ comme échelle de température, égale à la surchauffe paroi par rapport à la température liquide $T_{w}-T_{1}$. Le « quenching » étant instationnaire, nous n'avons pas introduit d'échelle de temps, $t$ sera alors normé par $D / V_{k}$. Nous aurions également $\mathrm{pu}$ choisir comme vitesse caractéristique la vitesse du front de remouillage pour prendre en compte l'instabilité principale du problème. Cependant, cette vitesse est très différente de celle de chaque phase, et nous ne pouvons la connaitre à priori. Concernant la pression, les frottements, et l'enthalpie totale, ils seront normés respectivement par $\rho_{k} V_{k}^{2}, \mu_{k} V_{k} / D$ et $C p_{k} \Theta$.

$$
\begin{gathered}
\frac{\partial x_{k}}{\partial t^{*}}+\frac{\partial x_{k}^{2} / \alpha_{k}}{\partial z^{*}}-\frac{V_{i}}{V_{k}} \frac{\partial x_{k}}{\partial z^{*}}+\alpha_{k} \frac{\partial p_{k}^{*}}{\partial z^{*}}-\frac{\alpha_{k}}{F r_{k}} \\
-\frac{1}{R e_{k}}\left(\tau_{w, k}^{*} \frac{D P_{w, k}}{A}-\tau_{i, k}^{*} \frac{D P_{i, k}}{A}\right)=0 \\
\frac{\partial h_{k}^{*} \alpha_{k}}{\partial t^{*}}+\frac{\partial h_{k}^{*} x_{k}}{\partial z^{*}}-h_{k, s a t}^{*} \frac{\partial x_{k}}{\partial z^{*}}+E c k_{k} \alpha_{k} \frac{\partial p_{k}^{*}}{\partial t^{*}}-\frac{E c k_{k}}{F r_{k}} x_{k} \\
-\frac{1}{P e_{k}}\left(q_{w, k}^{*} \frac{D P_{w, k}}{A}-q_{i, k}^{*} \frac{D P_{i, k}}{A}\right)=0 \\
\frac{J a_{1}}{P e_{1}} q_{i, 1}^{*}+\frac{J a_{2}}{P e_{2}} q_{i, 2}^{*}-\frac{A}{D P_{i}} \frac{\partial x_{2}}{\partial z^{*}}=0
\end{gathered}
$$

Dans les équations (7), (8) et (9), les formes adimensionnelles des équations de conservation de la quantité de mouvement, de l'enthalpie et du bilan interfacial d'enthalpie, on peut recenser les nombres adimensionnels caractéristiques : les Reynolds $\frac{V_{k} D}{\nu_{k}}$, Froude $\frac{V_{k}^{2}}{g D}$, Eckert $\frac{V_{k}^{2}}{C p_{k} \Theta}$, Jacob $\frac{C p_{k} \Theta}{\Delta h_{1,2}}$, et Peclet $\frac{G D C p_{k}}{\lambda_{k}}$. En micro pesanteur, dans un moteur-fusée, le nombre de Froude est très grand $\left(10\right.$ à $\left.10^{5}\right)$, et pour l'ordre de grandeur de vitesse liquide de $1 \mathrm{~m} . \mathrm{s}^{-1}$, le nombre d'Eckert est très petit $\left(10^{-5}\right.$ à 0,1$)$. Ainsi, la seule condition pour le respect de l'ordre de grandeur de ces deux nombres sera la conduite des expériences en micro pesanteur, par exemple à bord de vols paraboliques $\left(10^{-2} \mathrm{~g}\right)$.

Afin de respecter l'égalité des autres nombres, le diamètre de la section d'essai, et le débit massique seront fixés en considérant l'égalité du nombre de Reynolds $\left(10^{2}\right.$ à $\left.10^{5}\right)$. Le choix du fluide est fait en considérant le nombre de Peclet $\left(10^{2}\right.$ à $\left.10^{6}\right)$. Le nombre de $J a$ cob pouvant être décomposé en un impact de la surchauffe paroi et un impact du sous refroidissement ( $J a_{k}=$ $\left.\frac{C p_{k}\left(T_{w}-T_{s}\right)}{\Delta h_{1,2}}+\frac{C p_{k}\left(T_{s}-T_{1}\right)}{\Delta h_{1,2}}\right)$, ces deux paramètres seront fixés par l'égalité de ce nombre ( 0 à 2 pour l'oxygène, 0 à 8 pour l'hydrogène). Évidemment, aucun fluide ne permet de respecter l'égalité de chaque nombre, mais certains réfrigérants comme le HFE7000 ou le FC72 peuvent permettre d'obtenir une similitude suffisante avec les écoulements cryogéniques présents lors de mise en froid de moteurs-fusée.

Ainsi, des essais qualitatifs ont été menés avec du FC72 sur l'installation MICROBO (Sect. 4). Ceux -ci ne nous permettent pas de respecter la similitude de l'ensemble des configurations réelles, en raison des faibles valeurs de Reynolds $\left(R e_{1}<7000\right)$, et des larges valeurs de Jacob $\left(J a_{1}>0,3\right)$ dues à un fort sous refroidissement. Une nouvelle installation expérimentale est donc en cours de développement à l'IMFT de Toulouse. Celle-ci consiste en une boucle diphasique proche de celle de MICROBO, décrite en reference [24]. La section d'essai sera un tube d'inox de $1 \mathrm{~cm}$ de diamètre et de $10 \mathrm{~cm}$ de long, mis en froid par un écoulement de NovecHFE7000(3M). Le choix de ce fluide a été fait car il permet la mise en place d'une mesure locale capacitive de taux de vide, basée sur le contraste de permittivité des deux phases. De plus, le point d'ébullition de ce fluide est bas (34 degrés Celsius à 1 bar), et ses propriétés physiques permettent de mieux respecter la similarité en Peclet. Des essais de «quenching » seront menés à bord d'un Airbus A300 «ZeroG » en octobre 2009.

\section{Résultats expérimentaux obtenus avec MICROBO}

Des essais qualitatifs ont été menés sur l'installation MICROBO, conçue par l'ENEA de Rome, durant 87 paraboles. Les sections d'essais consistaient en des tubes de pyrex, de $16 \mathrm{~cm}$ de long et d'un diamètre interne allant de $2 \mathrm{~mm}$ à $6 \mathrm{~mm}$. La pression, le débit, et le sous refroidissement ont varié de 1,6 bar à $3 \mathrm{bar}$, de $60 \mathrm{~kg} \cdot \mathrm{m}^{2} \cdot \mathrm{s}^{-1}$ à $450 \mathrm{~kg} \cdot \mathrm{m}^{2} \cdot \mathrm{s}^{-1}$, et de $-20 \mathrm{~K}$ à $-50 \mathrm{~K}$ respectivement. Les essais correspondants ont été menés au sol, en configuration ascendante et descendante.

\subsection{Configuration de l'écoulement}

Ces tests ont montré une forte différence entre les essais en écoulement descendant et ascendant ou en micro 


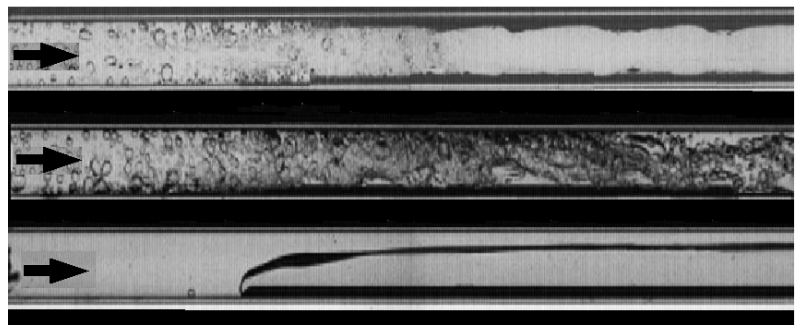

Fig. 2. Écoulements observés pour $G=180 \mathrm{~kg} \cdot \mathrm{m}^{2} \cdot \mathrm{s}^{-1}, D=$ $6 \mathrm{~mm}$ et un sous refroidissement de $35 \mathrm{~K}$. De haut en bas : $0 g$, ascendant, descendant.

pesanteur. En $0 g$, l'écoulement semble symétrique et le cœur liquide stable (Fig. 2). En ascendant, le coeur liquide est plus large en raison de son poids, la couche vapeur est alors plus mince et la vitesse de celle-ci plus élevée. Donc, l'interface liquide/vapeur est moins stable en raison du gradient de vitesse. En descendant, des bulles de vapeur peuvent remonter de manière asymétrique, voir se stabiliser, en raison de la poussée d'Archimède. En micro pesanteur, le coeur liquide étant plus stable, il peut s'amenuiser sans se déstabiliser. Cette configuration unique à la micro pesanteur a été observée auparavant [3] et est appelée «écoulement filamentaire ».

La configuration en $0 g$ étant plus proche de celle ascendante que de celle descendante, nous comparerons les résultats obtenus lors de vols paraboliques uniquement avec les essais correspondants ascendants.

\subsection{Flux thermique lors de l'ébullition en film et température de remouillage}

La seule mesure de température effectuée est celle à l'extérieur de la paroi du tube. Le flux thermique $Q(z, t)$ est donc défini en fonction de la variation temporelle de cette mesure, en supposant un gradient temporel homogène dans l'épaisseur de la paroi (Éq. (10)). La température interne $T_{\text {int }}(z, t)$ est alors obtenue en supposant un état quasi stationnaire (Éq. (11)).

$$
\begin{aligned}
Q(z, t) & \approx \frac{\rho_{w} A_{w} C p_{w}}{P_{w}} \frac{\partial T_{w}(z, t)}{\partial t} \\
T_{\text {int }}(z, t) & =\frac{1}{2-d}\left(Q(z, t) \frac{d}{\lambda_{w}}-(d+e-2) T_{w}(z, t)\right)
\end{aligned}
$$

Sur la figure 3 , on peut observer une différence importante, de l'ordre de $20 \mathrm{~K}$, entre les températures externes et internes. Cela est dû à la forte inertie thermique du tube de pyrex et il est alors indispensable de définir la température de remouillage d'après le profil interne. Cette dernière est obtenue en prenant l'intersection des pentes de l'ébullition en film et de l'ébullition de transition.

Sur la figure 3, nous pouvons observer également des courbes d'ébullition typiques en micro pesanteur et en écoulement ascendant, toute chose étant égale par ailleurs. Comme cela a été observé précédemment, le flux thermique lors de l'ébullition en film diminue en $0 g[14,15]$. Dans cette configuration, le film de vapeur est plus large et la vitesse de celle-ci est donc plus faible. L'échange entre la paroi et le liquide se retrouve donc diminué, ainsi que l'échange avec la vapeur par convection forcée. À la fin de la mise en froid, le régime de convection forcée monophasique liquide est logiquement non influencé par le niveau de gravité. Concernant le flux critique, et le flux lors de l'ébullition en film, aucune tendance précise ne peut être mise en avant. En effet, après le remouillage, l'échange thermique est beaucoup plus rapide, mettant à mal notre hypothèse de gradient temporel de la temperature homogène, rendant les incertitudes sur ces résultats trop importantes.

Sur la figure 4, on peut observer que le flux semble augmenter avec le sous refroidissement, tout en restant plus faible en micro pesanteur. Au vu des incertitudes, aucun impact précis ne peut être obtenu, mais cette tendance avec le sous refroidissement est cohérente avec les modèles existants [17].

Nous pouvons également observer sur les figures 3 et 4 , toujours sans pouvoir tirer de conclusions nettes, que la température de remouillage semble augmenter en micro pesanteur. Cela pourrait être expliqué par l'élargissement de la couche de vapeur dans le régime d'ébullition en film. En effet, le cœur liquide étant plus fin, il est plus rapide. Les gouttes de liquide arrachées vont alors impacter la paroi à une vitesse plus importante qu'en $1 \mathrm{~g}$, et vont avoir tendance à rebondir et remouiller plus difficilement la paroi, comme prédit par le modèle de remouillage de Iloege [2]. Sur la figure 4, la température de remouillage semble augmenter avec le sous refroidissement, en accord avec certains modèles existants (Éq. (2)), pour les deux niveaux de gravité.

Sur la figure 5 il est possible de déterminer un impact quantitatif du nombre de Froude sur la température de remouillage et le coefficient d'échange, celui-ci étant calculé comme étant $Q_{f b} /\left(T_{i n t}-T_{l}\right)$. Le nombre de Froude est obtenu avec la moyenne quadratique (RMS) de l'accélération $\left(\sqrt{\left\langle g^{2}\right\rangle}\right)$, égale à $9,81 \mathrm{~m} . \mathrm{s}^{-2}$ pour les essais ascendants, et de l'ordre de $1,5 \times 9,81 \times 10^{-2} \mathrm{~m} . \mathrm{s}^{-2}$ pour les essais en apesanteur. Ici, il semble également que le flux soit plus faible en apesanteur, et la température de remouillage plus importante. L'hypothèse annoncée, qui justifie ces tendances par l'élargissement de la couche de vapeur, et donc l'accélération du liquide, lors de l'ébullition en film en gravité réduite est cohérente avec les tendances en Froude. En effet, la température de remouillage semble augmenter avec ce nombre, et donc avec la vitesse. En revanche, il est difficile de quantifier un impact de $F r$ concernant le coefficient d'échange, en raison des incertitudes sur les résultats. Pour que notre hypothèse soit cohérente avec la figure 4 , il faudrait que le flux diminue avec $\mathrm{Fr}$.

\section{Conclusion et perspectives}

Le sujet de l'étude détaillée dans cet article était d'établir le besoin expérimental pour l'obtention de lois 

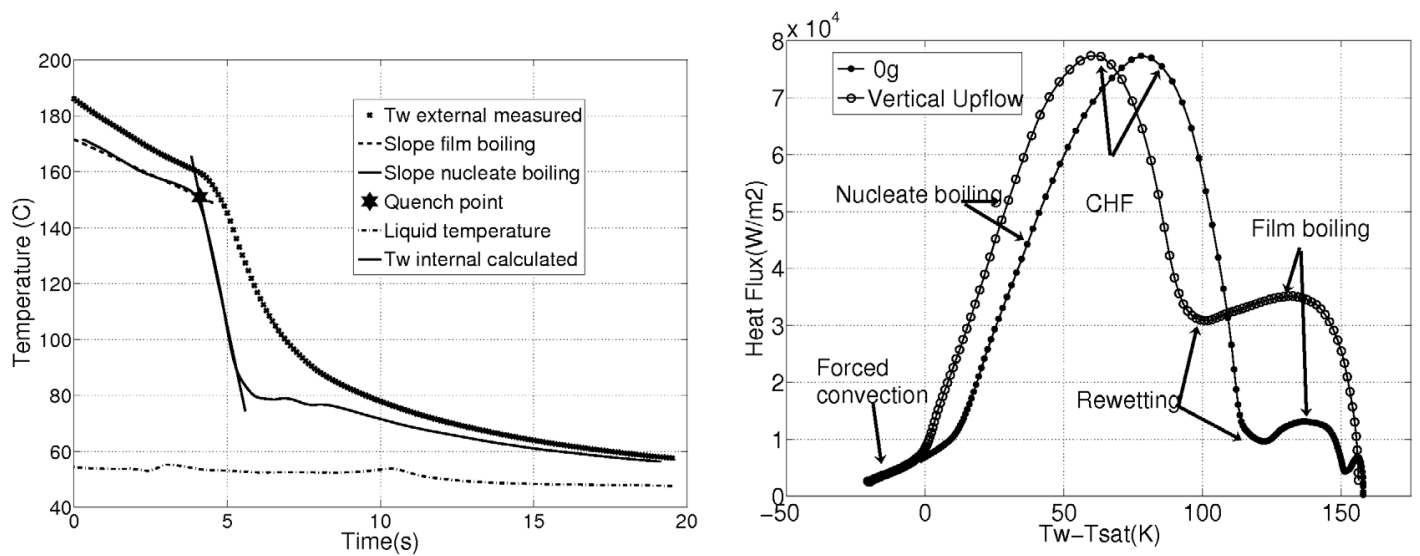

Fig. 3. A gauche : température externe mesurée, et interne calculée en $0 g$. A droite : flux thermique en fonction de la surchauffe paroi. $P=3$ bar, $G=160 \mathrm{~kg} \cdot \mathrm{m}^{-2} \cdot \mathrm{s}^{-1}, D=4 \mathrm{~mm}$, sous refroidissement de $25 \mathrm{~K}$.
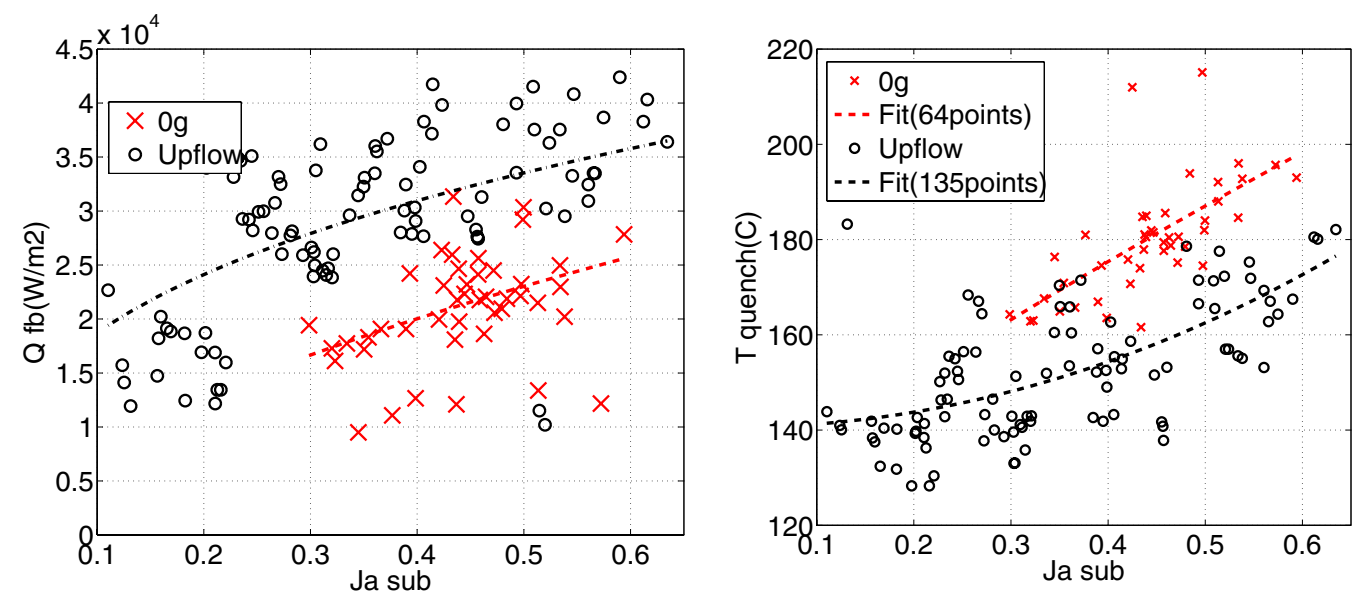

Fig. 4. Flux lors de l'ébullition en film (gauche) et température de remouillage (droite) en $0 g$ et $1 g$ ascendant en fonction du $J a c o b$ de sous refroidissement $C p_{1}\left(T_{s}-T_{1}\right) / \Delta h_{1,2}$. Les tendances sont basées sur un lissage de l'ensemble des données.
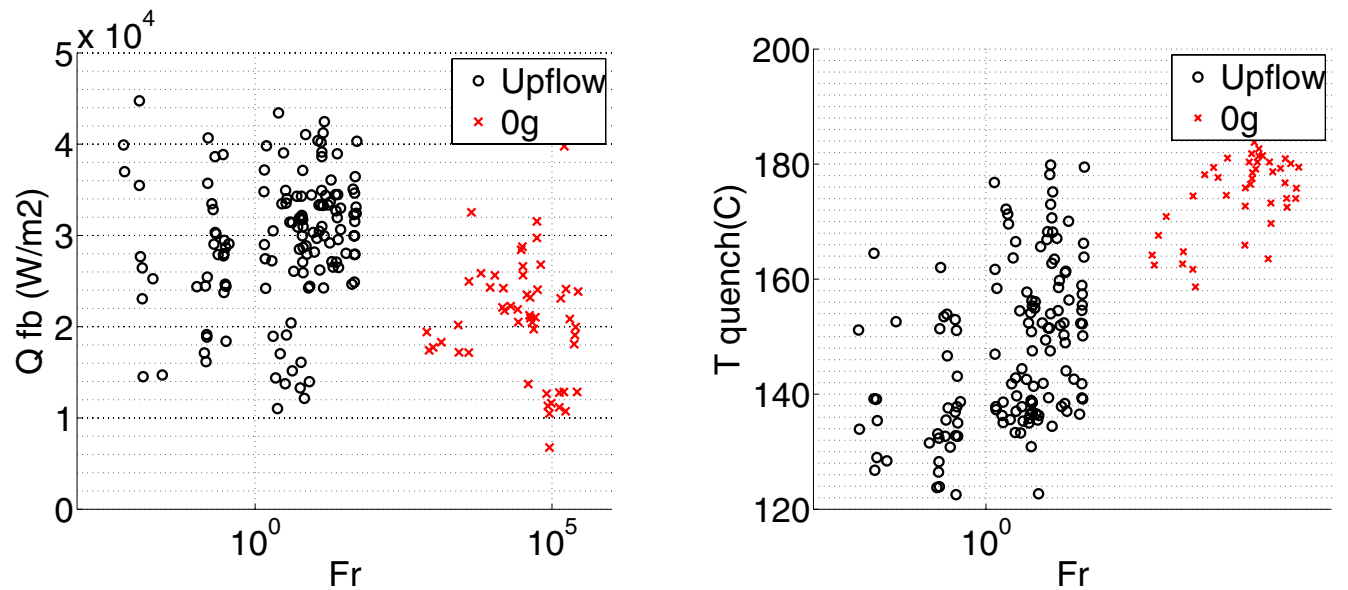

Fig. 5. Coefficient d'échange lors de l'ébullition en film (gauche) et température de remouillage (droite) en $0 \mathrm{~g}$ et $1 g$ ascendant en fonction du Froude d'entrée $V_{1}^{2} / g D$. 
de fermetures applicables aux écoulements de «quenching » en micro pesanteur. Des critères de similitudes ont été définis, en se basant sur l'adimensionnalisation des équations pertinentes. Une analyse de la littérature a mis en avant le manque de modèles applicables en $0 g$. Un recensement des expériences de « quenching » menées a montré que certaines tendances ont pu être mises en avant, mais pas de conclusions précises.

Des essais qualitatifs de «quenching » ont été menés durant 87 paraboles à bord d'un Airbus A300 «ZeroG ». Conformément aux précédentes observations, les résultats montrent une diminution du transfert thermique lors de l'ébullition en film, et une augmentation de la température de remouillage en gravité réduite. Cela pourrait être expliqué par l'élargissement de la couche de vapeur lors de l'ébullition en film.

Des essais complémentaires doivent être menés sur une nouvelle installation, en développement, lors de la campagne de vols paraboliques d'octobre 2009 afin d'obtenir des résultats quantitatifs.

Remerciements. Les auteurs souhaiteraient remercier SNECMA Moteur, le Centre national d'études spatiales (CNES) et l'Agence Spaciale Européenne (ESA) pour le support de cette étude.

\section{Références}

[1] G.P. Purohit, V.K. Dhir, Subcooled film-boiling heat transfer from spheres, Nuclear Engineering and Design 47 (1978) 49-66

[2] O.C. Iloeje, A study of Wall Rewett and Heat Transfer in Dispersed Vertical Flow, Ph.D. Thesis, Massachusetts Inst. Technology, 1975

[3] F.G. Collins, B.N. Antar, Flow boiling during quench in low gravity environment, Microgravity science and technology, 1997

[4] J.A. Bromley, Heat transfer in stable film boiling, Chemical Engineering Progress, 1950

[5] B.P. Breen, Effect of diameter of horizontal tubes on film boiling, Chemical Engineering Progress 58 (1962) 67

[6] J.A. Bromley, Heat transfer in forced convection film boiling, Industrial Engineering Chemistry 45 (1953) 26392646

[7] P.J. Berenson, Film boiling heat transfer from a horizontal surface, J. Heat Transfer C 83 (1961) 351-356

[8] G.E. Dix, J.E. Leonard, K.H. Sun, Solar and nuclear heat transfer, AIChE Symp. Ser. (73/164) (1977) 7
[9] J.A. Clark, T.H.K. Frederking, Natural convection film boiling on a sphere, Advanced Cryogenic Engineering 8 (1962) 501-506

[10] I. Shai, Y. Barnea, E. Elias, Flow heat transfer regimes during quenching of hot surfaces, Int. J. Heat Mass Transfer 37 (1994) 114-1453

[11] S.C. Cheng, N. Hammouda, D.C. Groeneveld, Two fluid modeling of inverted annular film boiling, Int. J. Heat Mass Transfer 40 (1997) 2655-2670

[12] B.N. Antar, C.J. Westbye, M. Kawaji, Boiling heat transfer in the quenching of a hot tube under microgravity, J. Thermophys. Heat Transfer 9 (1995)

[13] H. Otha, O. Kawanami, H. Azuma, Effect of gravity on cryogenic boiling heat transfer during tube quenching, Int. J. Heat Mass Transfer 50 (2007) 3490-3497

[14] J.J. Xu, Flow boiling heat transfer in the quenching of a hot surface under reduced gravity conditions, Ph.D. Thesis, University of Toronto, 1998

[15] M. Kawaji, Boiling heat transfer during quenching under microgravity, American Inst, Aeronautics Astronautics, 1996

[16] M. Silbergberg, C.F. Bampus, A.Norman, P. Spiegler, J. Hopenfeld, Onset of stable film boiling, and the foam limit, Int. J. Heat Mass Transfer 6 (1963) 987-989

[17] J.G. Collier, Heat transfer in the post burnout region and during quenching and reflooding, Handbook of Multiphase Systems, chapter VI, Hemisphère Publishing Corporation, 1982

[18] F.F. Simon, K.J. Baumeister, Leidenfrost temperature its correlation for liquid metals, cryogens, hydrocarbons and water, J. Heat Transfer 95 (1973) 166-173

[19] B. Panella, M. De Salve, Thermal-hydraulics of the precursory cooling during bottom reflooding, Multi-phase flow and heat transfer symposium-workshop, 1983

[20] J.J. Carbajo, A study on the rewetting temperature, Nuclear Engineering and Design 84 21-52 (1985)

[21] Y. Zimmels, M. Ben David, Y. Zvirin, Determination of the quench velocity and rewetting temperature of hot surfaces: formulation of a nonisothermal microscale hydrodynamic model, Phys. Rev. APS 59 (1999) 6687-6698

[22] J.N. Chung, K. Yuan, Y. Ji, Cryogenic chill down process under low flow rates, Int. J. Heat Mass Transfer 50 (2007) 4011-4022

[23] M. Kawaji, K. Adham-Khodaparast, J.J. Xu, Flow film boiling collapse and surface rewetting in normal and reduced gravity conditions, Int. J. Heat Mass Transfer 38 (1995) 2749-2760

[24] G. Zummo, G.P. Celata, M. Cumo, Quenching experiments at reduced gravity, Invited Lecture, 3rd Intern. Symposium on Physical Sciences in Space, 2007 